\title{
Binary Black Hole Mergers within the LIGO Horizon: Statistical Properties and prospects for detecting Electromagnetic Counterparts
}

\author{
Rosalba Perna ${ }^{1}$, Martyna Chruslinska ${ }^{2}$, Alessandra Corsi $^{3}$, Krzysztof Belczynski $^{4}$ \\ 1 Department of Physics and Astronomy, Stony Brook University, Stony Brook, NY, USA \\ 2 Warsaw University Observatory, Al Ujazdowskie 4, 00-478 Warszawa, Poland \\ 3 Department of Physics, Texas Tech University, Box 41051, Lubbock, TX 79409-1051, USA \\ 4 Nicolaus Copernicus Astronomical Centre, Polish Academy of Sciences, ul. Bartycka 18, 00-716 Warsaw, Poland
}

19 April 2018

\begin{abstract}
Binary black holes (BBHs) are one of the endpoints of isolated binary evolution, and their mergers a leading channel for gravitational wave events. Here, using the evolutionary code STARTRACK, we study the statistical properties of the BBH population from isolated binary evolution for a range of progenitor star metallicities and $\mathrm{BH}$ natal kicks. We compute the mass function and the distribution of the primary BH spin $a$ as a result of mass accretion during the binary evolution, and find that this is not an efficient process to spin up BHs, producing an increase by at most $a \sim 0.2-0.3$ for very low natal BH spins. We further compute the distribution of merger sites within the host galaxy, after tracking the motion of the binaries in the potentials of a massive spiral, a massive elliptical, and a dwarf galaxy. We find that a fraction of $70-90 \%$ of mergers in massive galaxies and of $40-60 \%$ in dwarfs (range mostly sensitive to the natal kicks) is expected to occur inside of their hosts. The number density distribution at the merger sites further allows us to estimate the broadband luminosity distribution that $\mathrm{BBH}$ mergers would produce, if associated with a kinetic energy release in an outflow, which, as a reference, we assume at the level inferred for the Fermi GBM counterpart to GW150914, with the understanding that current limits from the O1 and $\mathrm{O} 2$ runs would require such emission to be produced within a jet of angular size within $\lesssim 50^{\circ}$.
\end{abstract}

Key words: gravitational waves — stars: black holes — gamma -ray burst: general

\section{INTRODUCTION}

The LIGO discovery of gravitational waves (GWs) from BBH mergers (Abbott et al. 2016d,b, 2017) has opened a new window onto the universe, providing a definite confirmation that binary BHs exist, and merge with a local rate in the range $\sim 12-213 \mathrm{Gpc}^{-3} \mathrm{yr}^{-1}$ (Abbott et al. 2016f, 2017).

The properties of the LIGO-discovered $\mathrm{BHs}$, and in particular the large masses and relatively low spins, have triggered numerous investigations aimed at identifying the possible formation channels of BHs with such properties. Three main mechanisms have been considered: Classical isolated binary evolution, dynamical formation, and chemically homogeneous evolution in tidally distorted binary stars. Within the classical isolated binary evolution scenario, the binary system undergoes a common envelope (CE) ejection, or a non-conservative mass transfer (e.g. Tutukov \& Yun- gelson 1993; Kalogera et al. 2007; Postnov \& Yungelson 2014). Dynamical formation involves dynamical interactions in dense star clusters (Sigurdsson \& Hernquist 1993; Downing et al. 2010, 2011; Antonini et al. 2016; Rodriguez, Chatterjee \& Rasio 2016; Rodriguez et al. 2017). For example, isolated $\mathrm{BHs}$ can acquire a companion via 3-body exchanges and binary-mediated interactions, which tend to result in the ejections of the lightest BH. Finally, for a chemically homogeneous evolution in tidally distorted binary stars, strong internal mixing occurs as a result of massive stars being in near contact binaries (de Mink et al. 2009; de Mink \& Mandel 2016; Mandel \& de Mink 2016; Marchant et al. 2016).

During the first (O1) and second (O2) observing runs of advanced LIGO, a massive follow-up campaign in search for electromagnetic (EM) counterparts to GWs has been undertaken (e.g. Abbott et al. 2016c; Copperwheat et al. 2016; Cowperthwaite et al. 2016; Evans et al. 2016; Kasli- 
wal et al. 2016; Morokuma et al. 2016; Palliyaguru et al. 2016; Savchenko et al. 2016; Smartt et al. 2016b,a; Bhalerao et al. 2017; Corsi et al. 2017; Kawai et al. 2017; Racusin et al. 2017; Savchenko et al. 2017). A combined GW-EM detection would help break GW parameter degeneracies (see e.g. Pankow et al. 2017), it would enable measurement of the source redshift (Schutz 1986; Abbott et al. 2017a), and help constrain the formation channel of the $\mathrm{BBH}$ binary by probing the environment in which the merger occurred (e.g. dynamical formation (Rodriguez, Chatterjee \& Rasio 2016) is likely to occur in dense star clusters, while the classical isolated binary evolution scenario (Kalogera et al. 2007) in typical field galaxies; on the other hand, $\mathrm{BBH}$ evolution in an accretion disk around a supermassive black hole (Bartos et al. 2017) will be found in the vicinity of AGNs).

To date, most searches for EM counterparts to the LIGO detections have yielded negative results. Possible exceptions are the potential $\gamma$-ray counterparts to GW150914 and GW170104 identified by the Fermi (Connaughton et al. 2016, but see Greiner et al. 2016) and AGILE (Verrecchia et al. 2017) satellites, respectively. While in NS-NS and NS$\mathrm{BH}$ mergers EM emission is a natural outcome of the circularization, and subsequent accretion, of some mass from the tidally disrupted NS, in the case of BBH mergers no EM emission is generally expected. Thus, the tentative $\gamma$-ray detections by the Fermi and AGILE satellites have received considerable attention, and spurred several ideas and discussions on the possible presence of EM counterparts to $\mathrm{BBH}$ mergers (Perna, Lazzati \& Giacomazzo 2016; Zhang 2016; Loeb 2016; Lyutikov 2016; Fraschetti 2016; Woosley 2016; Murase et al. 2016; Liebling \& Palenzuela 2016; Li et al. 2016; Bartos et al. 2017; Janiuk et al. 2017; Dai, McKinney \& Miller 2017; Stone, Metzger \& Haiman 2017; Ioka et al. 2017; de Mink \& King 2017; Kimura, Murase \& Mészáros 2017).

Given the large localization areas of GW events detected by LIGO (Abbott et al. 2016e), being able to design an EM follow-up strategy which optimizes the chances of detection is of paramount importance. The very first step toward this goal is gaining a better theoretical understanding of the relation between $\mathrm{BBH}$ binary properties, their merger sites, and the expected brightness of their potential EM counterparts. However, to date, there exists no specific prediction as to the most likely sites of $\mathrm{BBH}$ mergers within their host galaxies, and hence as to what afterglow brightness could be expected if these mergers were to give rise to explosive, gamma-ray burst (GRB)-like counterparts.

In light of the above, goal of this paper is to make statistical predictions for the observable properties of $\mathrm{BBH}$ mergers, and their possible EM counterparts, within the specific evolutionary model of isolated binary evolution, and within the standard GRB afterglow model (e.g., Sari, Piran \& Narayan 1998) for the production of EM counterparts at various wavelenghts (see also Yamazaki, Asano \& Ohira 2016). We build upon previous work of our group addressed at NS-NS and NS-BH mergers (Perna \& Belczynski 2002; Belczynski et al. 2006). Using the code StarTrack (Belczynski et al. 2008), we predict: (i) the BBH chirp and total mass distribution for several combinations of model assumptions and metallicities; (ii) the distribution of merger sites within their host galaxies; (iii) the increase in the spin distribution of the primary $\mathrm{BH}$ due to accretion from the secondary for several values of the initial spin; (iv) the expected afterglow luminosities in the X-ray, optical, and radio bands. For this last calculation, we take as a prototype the energetics $\left(E \sim 10^{49} \mathrm{ergs}\right)$ inferred for the possible $\gamma$-ray counterpart to GW150914 (Connaughton et al. 2016). While this detection may be spurious (Greiner et al. 2016), we use it here simply as a potential calibration point that may need to be changed when (and if) a secure BBH EM counterpart is detected. Hence, we present our results in a way such that they can be easily rescaled to different energy values. We emphasize our agnostic view as to whether EM counterparts to $\mathrm{BBH}$ mergers can exist. In this light, our main aim for performing this statistical computation of EM counterparts is that of providing a test for ideas predicting the possibility of an impulsive kinetic energy release associated with BBH mergers. More specifically, our calculations are meant to provide the link between the ideas mentioned above (and discussed in more detail in Sec. 4), and the panchromatic observational campaigns that have (and will) follow GWdetected $\mathrm{BBH}$ mergers.

The paper is organized as follows: the description of the galactic potentials used is detailed in Sec. 2, while Sec. 3 describes the population synthesis calculation, our model assumptions, and the initial conditions for the simulations. Sec. 4 presents the computation of the afterglow spectrum under the assumption that merger events drive a shock in the interstellar medium. The results of our calculations are reported in Sec. 5, and include the distributions for the chirp and total mass function, the spin distribution of the primary $\mathrm{BH}$, and the probability function for the location of merger events within their host galaxies, for several combinations of the metallicity and other relevant model parameters. The predicted electromagnetic luminosities are provided in three relevant bands. We summarize in Sec. 6 .

\section{GALAXY POTENTIAL MODELS}

We consider three galaxy models: a large, Milky Way-type spiral galaxy, a large elliptical, and a dwarf galaxy. The latter is motivated by recent suggestions (O'Shaughnessy et al. 2017) that dwarf galaxies may overabundantly produce compact binary mergers, and particularly binary BHs. The type of galaxy is important for two reasons: (i) its potential determines the motion of the binaries, for given initial speeds and locations; (ii) its medium density (in magnitude and spatial distribution) influences the brightness of potential EM counterparts (afterglows). We follow the motion of the $\mathrm{BBH}$ binaries in the model galaxies from their formation time until the merger (for those which merge within a Hubble time), taking into account the kick velocities gained at the formation of each compact object.

The model spiral galaxy consists of a disc and a bulge described by the Miyamoto \& Nagai (1975) type potential:

$$
\Phi_{M N}=-\frac{G M}{\sqrt{x^{2}+y^{2}+\left(a+\sqrt{z^{2}+b^{2}}\right)^{2}}}
$$

and a dark matter halo described by the Paczynski (1990) 
potential,

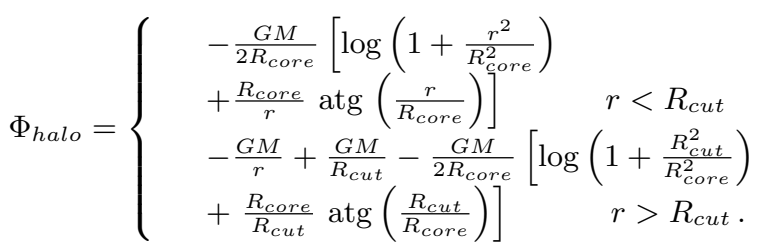

Our model elliptical galaxy consists of a bulge described by the (Hernquist 1990) type potential:

$$
\Phi_{H}=-\frac{G M_{E}}{r+a_{E}}
$$

and a halo with potential given by Eq. (2).

The parameters of the spiral galaxy potential are taken to be the same as those of the Milky Way: for the disc, $\mathrm{a}=$ $4.2 \mathrm{kpc}, \mathrm{b}=0.198 \mathrm{kpc}, \mathrm{M}=8.78 \times 10^{10} \mathrm{M}_{\odot}$; for the bulge, $\mathrm{a}=0, \mathrm{~b}=0.277 \mathrm{kpc}, \mathrm{M}=1.2 \times 10^{10} \mathrm{M}_{\odot}$; and for the halo, $\mathrm{M}_{\text {halo }}=5 \times 10^{10} \mathrm{M}_{\odot}, \mathrm{R}_{\text {core }}=6 \mathrm{kpc}, \mathrm{R}_{\text {cut }}=100 \mathrm{kpc}$. For the elliptical galaxy we assume a mass of $M_{E}=5 \times 10^{11} M_{\odot}$ and a scale factor of $\mathrm{a}_{\mathrm{E}}=5 \mathrm{kpc}$, while the halo parameters are assumed to be the same as those for the spiral galaxy. The fraction of mass in gas is assumed to be $f_{\text {gas }}=0.5$ for the bulge and disk, and $f_{\text {gas }}=\Omega_{b} / \Omega=0.04$ for the halo (Bahcall et al. 1999).

For the case of the spiral galaxy, binaries are initially placed on circular orbits in the disc. We adopt the distribution of stars within the disc of a spiral galaxy after Paczynski (1990):

$$
P(R, z) d R d z=P(R) d R p(z) d z
$$

where:

$$
\begin{array}{r}
P(R) d R=a_{R} e^{-R / R_{\exp }} \frac{R}{R_{\text {exp }}^{2}} d R \\
R=\sqrt{x^{2}+y^{2}} \\
a_{R}=\left[1-e^{-R_{\max / R_{\exp }}}\left(1+\frac{R_{\max }}{R_{\text {exp }}}\right)\right]^{-1}
\end{array}
$$

and

$$
p(z) d z=e^{-z / z_{\exp }} \frac{1}{z_{\text {exp }}} d z,
$$

with $R_{\exp }=4.5 \mathrm{kpc}, \mathrm{R}_{\max }=20 \mathrm{kpc}$, and $\mathrm{z}_{\exp }=75 \mathrm{pc}$.

In the case of the elliptical galaxy, binaries are placed in the galactic bulge with random orientation of the orbital angular momentum, and with a mass density corresponding to the Hernquist potential, $\rho(r)=\left(M_{E} / 2 \pi\right) a_{E} r^{-1}\left(a_{E}+r\right)^{-3}$.

To model the dwarf galaxy, we take as a proxy a small spiral galaxy, of total mass (disk + halo + bulge) $M_{\text {gal }}=$ $1.5 \times 10^{9} M_{\odot}$. Each individual mass component is rescaled to $0.1 \%$ of the corresponding one for the large spiral, while the spatial scale parameters of the potential are correspondingly rescaled to $10 \%$.

\section{BBH POPULATION SYNTHESIS MODELS}

To obtain the population of double $\mathrm{BH}$ binaries, we use the StarTrack population synthesis code, described in detail in Belczynski, Kalogera \& Bulik (2002); Belczynski et al. (2008), with the updated treatment of the CE phase (as described in Dominik et al. 2012), and the wind mass loss
(Belczynski et al. 2010).

Note that in StarTrack we only account for BBH binaries formed within the classical isolated binary evolution scenario involving mass transfer episodes and/or CE evolution, as opposed to other possible formation channels, such as dynamical formation in dense stellar environments (e.g. Rodriguez, Chatterjee \& Rasio 2016), or chemically homogeneous evolution (e.g. de Mink \& Mandel 2016; Marchant et al. 2016).

\subsection{Initial Conditions and Model Parameters}

For binaries with periods $<3000$ days, we adopt the initial conditions as described in Sana et al. (2012), based on spectroscopic observations of Galactic O-type stars: Kroupa-like IMF (IMF Kroupa, Tout \& Gilmore 1993) with the powerlaw exponent modified to -2.3 for the stars of mass $M>$ $1.0 \mathrm{M}_{\odot}$, eccentricity distribution $f(e) \propto e^{-0.42}$ in the range $[0.0,1.0]$, period distribution $f(P) \propto[\log (\mathrm{P}) / \text { days }]^{-0.55}$ in the range $[0.15,3.48]$ and a flat binary mass ratio distribution $q=\mathrm{M}_{\mathrm{b}} / \mathrm{M}_{\mathrm{a}}$, within $[0.0,1.0]$. Wider systems cannot be reliably probed through spectroscopic observations (Sana et al. 2012). Here, based on the recent review of Duchêne \& Kraus (2013), for systems with initial periods of over 3000 days, we adopt a mass ratio distribution $f(q) \sim q^{\gamma}$ with $\gamma=-0.5$. There is no clear evidence for the period distribution to change considerably with periods increasing over 3000 days; we thus keep the rest of initial distributions of Sana et al. (2012) unchanged and extend the period distribution up to $\log [P /$ days $]=5.5$.

We study in depth three evolutionary models, chosen to give the largest spread in natal kicks, as detailed in Sec. 3.2. For clarity and consistency with other published works, we keep the notation consistent with the one adopted by Belczynski et al. (2016a), and hence we use the same names for the models that we study. More specifically, we will consider models M10 (standard model), M13 and M15. These are the equivalent of models M1, M3 and M5 studied by Belczynski et al. (2016b), except that they include the effect of Pair-instability Pulsation Supernovae (PPSN) and Pair-instability Supernovae (PSN) ${ }^{1}$.

The reference model (M10) has the minimum mass of the primary $\mathrm{M}_{\text {mina }}=20 \mathrm{M}_{\odot}$, and minimum mass of the secondary $\mathrm{M}_{\text {minb }}=15 \mathrm{M}_{\odot}$, while the maximum allowed mass for a main sequence star is $\mathrm{M}_{\max }=150 \mathrm{M}_{\odot}$. The other models (M13/M15) have a minimum mass of the primary equal to $\mathrm{M}_{\text {mina }}=5 \mathrm{M}_{\odot}$, a minimum mass of the secondary given by $\mathrm{M}_{\text {minb }}=3 \mathrm{M}_{\odot}$, and maximum allowed mass as in $\mathrm{M} 10\left(\mathrm{M}_{\max }=150 \mathrm{M}_{\odot}\right)$. For each model, we run three sets of metallicities: $\mathrm{Z}_{\odot}=0.02,10 \% \mathrm{Z}_{\odot}, 1 \% \mathrm{Z}_{\odot}$ (the contribution to the merging population is the biggest for small metallicities, see Belczynski et al. 2016c). Since the dependence of the remnant masses on metallicity is much stronger than the dependence on the of center-of-mass kicks,

\footnotetext{
${ }^{1}$ For the results presented here, the main difference between the models with and without PPSN/PSN is in the high mass BH range, $M_{\mathrm{BH}} \gtrsim 40 M_{\odot}$, hence only of relevance for the tail of the mass function of the $Z=1 \% Z_{\odot}$ model.
} 
which (as we explain in what follows) vary among the models M10/M13/M15, we show results at different metallicities only for the M1 (standard) model. For this model, $2 \times 10^{7}$ isolated binaries from the above mass range were simulated for each metallicity, starting from zero-age main sequence (ZAMS) stars. For models M13 and M15, we simulated $2 \times 10^{7}$ and $2 \times 10^{6}$ binaries at ZAMS, respectively. The number of BBH systems which merge within a Hubble time is, for model M10, 3254 at $Z_{\odot}, 205335$ at $10 \% \mathrm{Z}_{\odot}$ and 519782 at $1 \% \mathrm{Z}_{\odot}$, while it is 1406 for M13 and 435 for M15 (both calculated at $10 \% \mathrm{Z}_{\odot}$ only). Our number of simulated systems, ultimately limited by computational time, is comparable to, or larger than, what adopted in other similar studies (i.e. O'Shaughnessy, Kalogera \& Belczynski 2005; O'Shaughnessy et al. 2005; Perna \& Belczynski 2002). The properties of $\mathrm{BBH}$ systems that merge, in particular for our standard model M10 at $10 \% \mathrm{Z}_{\odot}$, appear statistically robust, as we verified by computing the resulting distributions by including only a subset of systems.

\section{$3.2 \quad$ Natal kicks}

Natal kicks play a fundamental role in determining the location of the merger sites within the host galaxies (and hence the medium density encountered by a potential GRB-driven shock). Models M10/M13/M15 make the following different assumptions for the natal kicks. In model M10, natal kicks gained by the newly formed $\mathrm{BH}$ during the supernova explosion are drawn from a Maxwellian distribution with the velocity dispersion $\sigma=265 \mathrm{kms}^{-1}$, as proposed by Hobbs et al. (2005) based on observations of proper motions of single, young pulsars, but their values are lowered proportionally to the amount of material falling back onto the compact object (see Eq. 16 of Fryer et al. 2012). This model also accounts for a Blaauw kick velocity (a few $\mathrm{km} / \mathrm{s}$ ), gained by the center of mass of the binary due to mass loss during supernova explosion (Blaauw 1961). The amount of fallback matter increases with the BH mass, hence more massive BHs receive smaller natal kicks. Moreover, the most massive ones $\left(\mathrm{M}_{\mathrm{BH}} \gtrsim 10 \mathrm{M}_{\odot}\right.$ at solar metallicity) form through direct collapse, with no supernova explosion, and thus receive no birth kick. On the other hand, in the 'pessimistic' model M13, $\mathrm{BHs}$ receive full natal kicks drawn from a Maxwellian with $\sigma=265 \mathrm{~km} \mathrm{~s}^{-1}$ (which leads to birth velocities of the individal components of $\sim 400 \mathrm{~km} / \mathrm{s}$ ), but this time the kicks are not decreased due to fallback. Finally, model M15 has intermediate kicks drawn from a Maxwellian distribution with the lower velocity dispersion of $\sigma=130 \mathrm{~km} \mathrm{~s}^{-1}$ (equivalent of the M15 model in Belczynski et al. 2016b).

In order to get a better sense of the role of the natal kicks in the results for the merger locations within the host galaxies that will be shown in Sec.5.1, Fig. 1 shows the $\mathrm{BBH}$ center of mass velocity distribution after the formation of the second $\mathrm{BH}$, for the three models under consideration and $Z=10 \% Z_{\odot}^{2}$ (but the metallicity has very little influence compared to the different model assumptions discussed above).

${ }^{2}$ We adopt $Z_{\odot}=0.02$ (Villante et al. 2014).

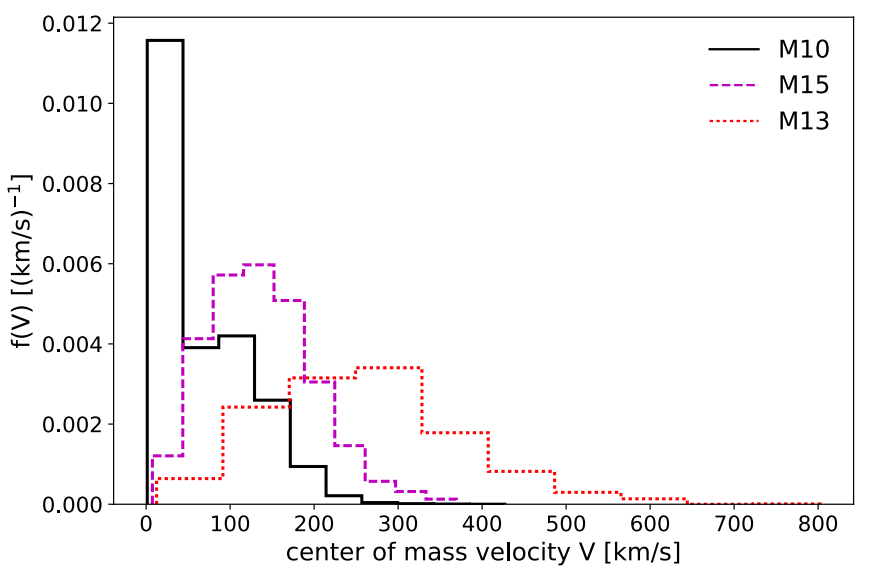

Figure 1. The BBH center of mass velocity distribution after the formation of the second $\mathrm{BH}$, for the three models under consideration.

\subsection{Binary Evolution}

In the following, we provide a brief outline of our simulations and the main assumptions behind them. More details can be found in Belczynski et al. (2016b) and references therein.

(i) Each binary system is followed from the ZAMS phase, and its evolution is assumed to proceed in isolation.

(ii) Binary parameters (mass of the primary, mass ratio, eccentricity, separation/period) are randomly drawn from the distributions described in the previous section.

(iii) The binary evolution includes simulations of tides, wind mass loss, and interaction between the components via mass transfer. These are modeled following the prescriptions detailed in Belczynski et al. (2008). Depending on the specific combination of parameters of each system, the binary may reach the point where the first compact object can form, or not (for instance, it can merge during the unstable mass transfer).

(iv) If there is a supernova, a certain amount of mass is lost instantaneously; the magnitude of the natal kick velocity is drawn from the assumed distribution (as described in the previous section), with a random direction. The magnitude of the natal kick, the amount of ejecta, and the parameters of the binary at the moment of the explosion determine whether the binary will be disrupted or not.

(v) The evolution of the binary is followed until the formation of the second compact object, or until the merger/disruption of the binary (if this happens before the formation of the double compact object system).

(vi) Given the final orbital parameters and the masses of each binary, we can then calculate the merger time due to gravitational radiation. Here we follow only the binaries which merge within the Hubble time.

\subsection{BH Spin Evolution due to accretion}

In our simulations BHs can accrete a significant amount of mass during the $\mathrm{CE}$ evolution. We assume that, during this phase, BHs accrete at $10 \%$ of the Bondi-Hoyle rate (Bondi 1952). Note that this accretion rate is somewhat uncertain, 

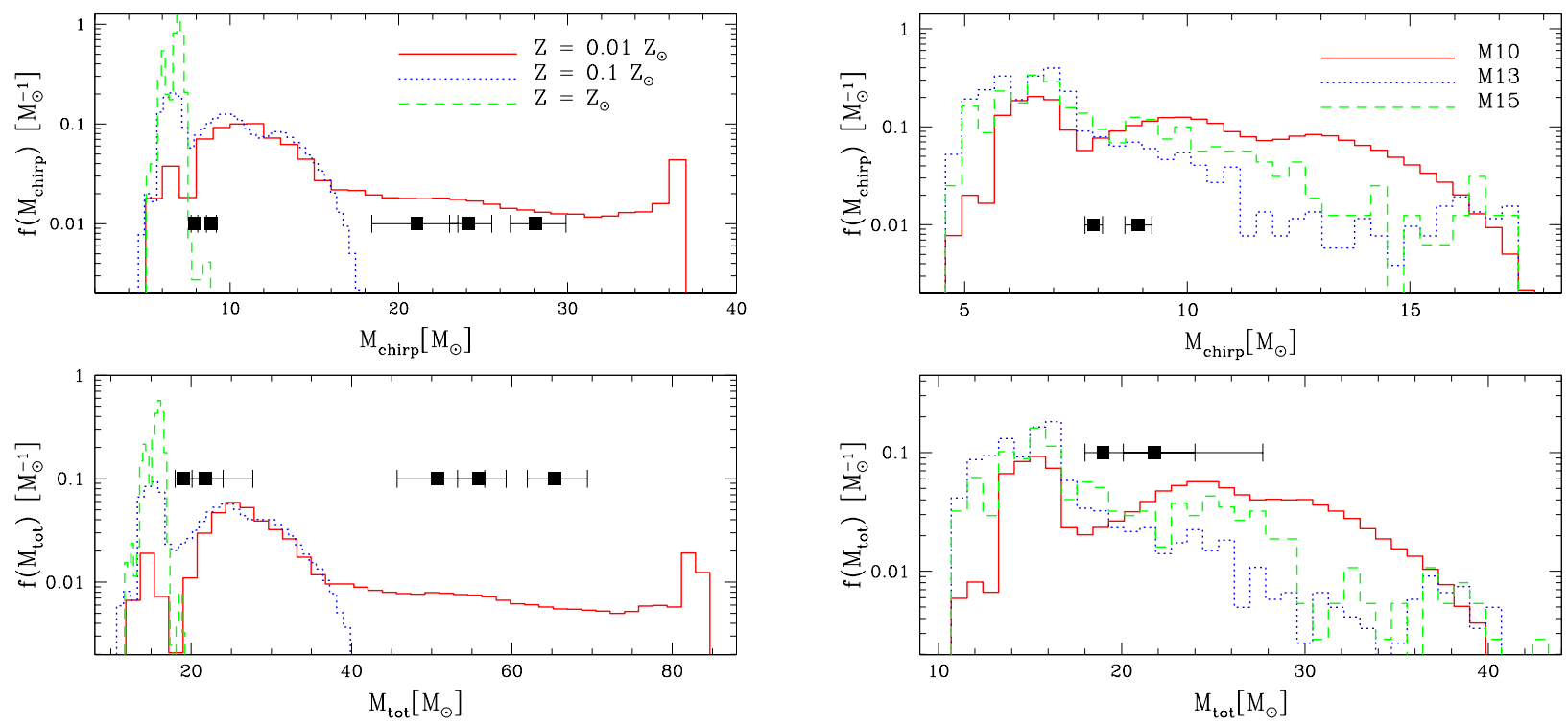

Figure 2. Left: The distribution of chirp masses (top panel) and of total masses (bottom panel) for the BH remnants (in the source frame) for three values of the metallicity, in the M10 model. Right: Same distributions as in the left panel, but for the 3 models studied here, at $10 \%$ of solar metallicity. Note that the dependence of the remnant mass fraction on metallicity is much stronger than variations in the mass function produced by different model assumptions for the natal kicks. The points with the errorbars show the measurements from the first two runs of LIGO (the height at which they are placed in the figures has clearly no meaning).

and recent simulations show that, when the density gradients across the $\mathrm{CE}$ are taken into account, the rate might be lower (e.g. MacLeod \& Ramirez-Ruiz 2015; MacLeod et al. 2017). As a reference with respect to previous related literature, our total amounts of accreted mass are close to the lower bounds of the calculations by O'Shaughnessy et al. (2005).

Our formalism for the computation of the change in $\mathrm{BH}$ spin as a result of $\mathrm{BH}$ accretion follows the literature (Shakura \& Sunyaev 1973; Thorne 1974). In particular, we adopt the formalism of Brown et al. (2000), also used by Belczynski et al. (2008).

The spin parameter of a $\mathrm{BH}$ of mass $M_{\mathrm{BH}}$ and angular momentum $J$ is defined as

$$
a=\frac{J c}{M_{\mathrm{BH}}^{2} G},
$$

where $G$ is the gravitational constant and $c$ the speed of light. If a $\mathrm{BH}$ with angular momentum $J_{\mathrm{i}}$ accretes an amount of material with rest mass $M_{\text {acc }}$, its new angular momentum will be given by

$$
J=J_{\mathrm{i}}+J_{\mathrm{acc}}
$$

where

$$
\begin{aligned}
J_{\mathrm{acc}} & =\left[\frac{R_{\mathrm{lso}}^{2}-\tilde{a} \sqrt{2 R_{\mathrm{Sch}} R_{\mathrm{lso}}}+\tilde{a}^{2}}{R_{\mathrm{lso}}\left(R_{\mathrm{lso}}^{2}-\frac{3}{2} R_{\mathrm{Sch}} R_{\mathrm{lso}}+\tilde{a} \sqrt{2 R_{\mathrm{Sch}} R_{\mathrm{lso}}}\right)^{1 / 2}}\right] \\
& \times c M_{\mathrm{acc}} \sqrt{\frac{R_{\mathrm{Sch}} R_{\mathrm{lso}}}{2}} .
\end{aligned}
$$

Here $R_{\mathrm{Sch}}=2 G M_{\mathrm{BH}} / c^{2}$ is the $\mathrm{BH}$ Schwarzschild radius, $\tilde{a} \equiv J_{\mathrm{i}} / M_{\mathrm{BH}} c=a_{\mathrm{i}}\left(G M_{\mathrm{BH}} / c^{2}\right)$, with $a_{\mathrm{i}}$ the initial BH spin; $R_{\text {lso }}$ is the radius of the last stable orbit

$$
R_{\mathrm{lso}}=\frac{R_{\mathrm{Sch}}}{2}\left\{3+r_{2}-\left[\left(3-r_{1}\right)\left(3+r_{1}+2 r_{2}\right)\right]^{1 / 2}\right\}
$$

with

$$
r_{1}=1+\left(1-\frac{4 \tilde{a}^{2}}{R_{\mathrm{Sch}}^{2}}\right)^{1 / 3}\left[\left(1+\frac{2 \tilde{a}}{R_{\mathrm{Sch}}}\right)^{1 / 3}+\left(1-\frac{2 \tilde{a}}{R_{\mathrm{Sch}}}\right)^{1 / 3}\right]
$$

and

$$
r_{2}=\left(3 \frac{4 \tilde{a}^{2}}{R_{\mathrm{Sch}}^{2}}+r_{1}^{2}\right)^{1 / 2} .
$$

Note that, as the $\mathrm{BH}$ accretes mass, its gravitational mass increases as

$$
M_{\mathrm{BH}, \mathrm{f}}=M_{\mathrm{BH}}+\frac{E}{c^{2}},
$$

where

$$
E=M_{\mathrm{acc}} c^{2}\left[\frac{R_{\mathrm{lso}}^{2}-R_{\mathrm{Sch}} R_{\mathrm{lso}}+\tilde{a} \sqrt{R_{\mathrm{Sch}} R_{\mathrm{lso}} / 2}}{R_{\mathrm{lso}}\left(R_{\mathrm{lso}}^{2}-\frac{3}{2} R_{\mathrm{Sch}} R_{\mathrm{lso}}+\tilde{a} \sqrt{2 R_{\mathrm{Sch}} R_{\mathrm{lso}}}\right)^{1 / 2}}\right] .
$$

\section{POSSIBLE EM COUNTERPARTS TO BBH MERGER EVENTS}

\subsection{Theoretical arguments and current observational constraints}

Whether $\mathrm{BBH}$ mergers can be accompanied by a release of energy in the form of a relativistic outflow, is a completely 
open question at the moment. This is true from both the observational and theoretical points of view. Observationally, the support comes from the tentative detection of a short $\gamma$-ray counterpart to GW150914 by Fermi GBM (Connaughton et al. 2016), and to GW170104 by AGILE (Verrecchia et al. 2017). The significance of the former detection has been challenged by Greiner et al. (2016), but more recently Connaughton et al. (2018) has argued against the criticism and reaffirmed their earlier conclusions. Independent analysis of the GBM data by another team (Bagoly et al. 2016) also found a potential counterpart near the time of GW150914.

Assuming that the detection was indeed real, then the question arises as to why none of the other $\mathrm{BBH}$ events in the LIGO runs $\mathrm{O} 1$ and $\mathrm{O} 2$ has had any detection, and what that implies in terms of constraints on the possible $\gamma$-ray energetics of these events. This question is currently the subject of an in-depth study by the Fermi team (V. Connaughton, private communication); however, a back-ofthe-envelope estimate can be made by simply using the distances to the BBH GW sources, and the Fermi sensitivity to short bursts, to derive the minimum observable luminosity for each of the sources. Adopting as a reference detection sensitivity to short bursts the least fluent burst detected by Fermi (Bhat et al. 2016), $F_{\min }=2.2 \times 10^{-8} \mathrm{erg} \mathrm{cm}^{-2} \mathrm{~s}^{-1}$, we derive a minimum detectable luminosity of $L_{\min } \sim$ $2.6 \times 10^{46} \mathrm{erg} \mathrm{s}^{-1} D_{100}^{2}$, where $D_{100}$ is the distance to the source in units of $100 \mathrm{Mpc}$. With the distances to the $\mathrm{BBH}$ GW events ranging between $340 \mathrm{Mpc}$ for GW170608 to $880 \mathrm{Mpc}$ for GW170104, we derive luminosity limits between $\sim 3 \times 10^{47} \mathrm{erg} \mathrm{s}^{-1}$ and $\sim 2 \times 10^{48} \mathrm{erg} \mathrm{s}^{-1}$. These upper limits on the luminosities are lower than what inferred for the potential Fermi counterpart to GW150914 ( $\left.L \sim 10^{49} \mathrm{erg} \mathrm{s}^{-1}\right)$. In order to assess their significance in constraining emission models, we need to estimate what is the actual detection probability of an event with the Fermi GBM. To this aim, we need to account for the fact that the GBM had about $60-80 \%$ coverage of the large LIGO annuli for the BBH mergers for which they had data, and that about $15 \%$ of the time they are turned off in the South Atlantic Anomaly. All together, this yields a roughly $50 \%$ chance of directly observing anything that happens anywhere in the sky (V. Connaughton, private communication). Considering a total of 6 events (including also the LIGO/Virgo trigger LVT151012 to be more conservative), the above estimates would then imply that about 3 out of the 6 events should have been detected by the Fermi GBM if the $\gamma$-ray emission was isotropic. A tentative detection of $\lesssim 1$ events would then imply that any putative $\gamma$-ray radiation would have to be produced within a jet of radial angular size $\theta_{\text {jet }}$ such that its beaming factor, $\left(1-\cot \theta_{\text {jet }}\right)$, is $\lesssim 1 / 3$. This yields $\theta_{\text {jet }} \lesssim 50^{\circ}$ (assuming two jets of the same size). To put this limit within the context of observations, the closest phenomenology we can rely on is that of the standard short GRBs. For these, a comprehensive analysis over a decade of data (Fong et al. 2015) showed that the median opening jet angle is $\sim 16^{\circ}$, which would be consistent with the current limits on non-detections of $\gamma$-rays from $\mathrm{BBH}$ mergers from geometric considerations alone. Therefore, the current $\gamma$-ray limits do not pose strong constraints on the presence of $\gamma$ ray radiation, unless there were reasons to believe that this had to be much more isotropic than what inferred for the short GRBs ${ }^{3}$.

From a theoretical point of view, the tentative $\gamma$-ray counterparts discussed above have led to a number of ideas being proposed for producing impulsive energy releases in association with a BBH merger. These include, among others (an extensive list can be found in the introduction), accretion from the envelope of a star whose core fragmented and collapsed into the two merging BHs (Loeb 2016, but see Woosley 2016), or a wind driven by a Poynting flux from charged BHs (Zhang 2016), or accretion from a fallback disk around one of the BHs (Perna, Lazzati \& Giacomazzo 2016). In this last model, the disk can survive for a long time as a dead disk, due to cooling and the suppression of viscosity once the magnetorotational instability is shut down; the disk is then revived and rapidly accretes during the final inspiral phase of the $\mathrm{BBH}$ merger. Note that a total energy reservoir of $\sim 10^{49}$ ergs as argued for GW150914 is something that could be easily produced in this astrophysical scenario. In fact, the stellar model used by Perna et al. (2016) was taken from a suite of standard pre-supernova profiles computed with the MESA code (Paxton et al. 2013) in an earlier work (Perna et al. 2014), and hence not fine-tuned for this particular event.

With severeal ideas in the literature for an energy reservoir connected to a $\mathrm{BBH}$ merger, the next question is whether a relativistic outflow can be driven. This issue has a long history of being investigated within the context of binary neutron stars mergers, where Magnetohydrodynamical (MHD) simulations in full General Relativity (GR) have given hints of a magnetically-driven outflow (Rezzolla et al. 2011; Ruiz et al. 2016; Kawamura et al. 2016; Ciolfi et al. 2017). Very recently, and of direct interest to this work, Khan et al. (2018) performed GRMHD simulations of disk accretion onto $\mathrm{BH}$ binaries, and observed collimated and magnetically-dominated outflows emerge in the disk funnel independently of the disk model. These important simulations lend support to the idea that, if there is a minidisk at the time of the BBH merger, then an outflow-induced shock can be driven into the medium, and generate an afterglowlike radiation similar to what observed in the case of the binary neutron star merger (Abbott et al. 2017b). Additionally, as pointed out by Murase et al. (2016), if a minidisk around the merging $\mathrm{BHs}$ were to exist, its thermal emission could be seen as an additional optical transient, lasting from hours to days. Shock heating of material surrounding the $\mathrm{BBH}$ site is also likely to produce broad-band radiation, from medium-energy X-rays to infrared (de Mink \& King 2017). These authors further discuss various mechanisms (in addition to the supernova fallback discussed above) by which the progenitor stars of $\mathrm{BHs}$ can shed mass during their evolution, including common-envelope ejection, eruptive mass-loss episodes, centrifugally shedding after spin up, mass shedding during non-conservative Roche-lobe overflow. The amount of mass available via these mechanisms can be

\footnotetext{
3 Note that in the case of binary NS mergers, an additional wide-angle, low-luminosity emission is expected from a cocoon produced when the relativistic jet propagates within the merger ejecta (e.g. Lazzati et al. 2017), but in the case of BBH mergers there would be no such ejecta for the jet to interact with.
} 
of several solar masses, and hence easily able to accomodate the requirement of $\sim 10^{-4} M_{\odot}$ set by the Fermi counterpart (see also Janiuk et al. (2017) for another binary evolutionary model leading to remnant mass at the time of the $\mathrm{BBH}$ merger). An abundant gas reservoir to tap energy from can also be of a different origin than the progenitor stars if the $\mathrm{BBH}$ is trapped in the inner region of the accretion disk of an Active Galactic Nuclei, in which case the BBH can accrete a significant amount of mass from the disk (Bartos et al. 2017; Stone, Metzger \& Haiman 2017).

Last, as an alternative to an energy reservoir due to matter as in the models discussed above, the $\mathrm{BBH}$ power could also be of electromagnetic nature due to a residual charge in the merging BHs (Zhang 2016; Liebling \& Palenzuela 2016). The simulations by Liebling \& Palenzuela (2016) showed that a BBH such as the one observed in GW150914 can satisfy the energy requirements of the Fermi GBM counterpart with a charge of $Q / M=10^{-4}$ assuming good radiative efficiency.

Whether any of these scenarios (or others) is realized in nature when two BHs merge is a question of great interest in astrophysics. For this reason, each LIGO detection of a BBH merger event has been followed by a massive observational campaign in a broad wavelength range. EM follow ups are expected to continue in future rounds of LIGO/Virgo runs.

The calculations in this paper reported so far allow us to proceed further and make an estimate of the broad-band radiation expected in association to high-velocity outflows propagating in the interstellar medium. This is because a shock would be a generic feature (Cavallo \& Rees 1978), rather independent of the specific details of the mechanism that produced the energy release. As the blastwave plows into the interstellar medium and slows down, it emits radiation over a broad-band energy range, the so-called afterglow. In the following (cfr. Sec.5.3), by taking a fully agnostic approach as to whether a (non-GW) energy release accompanies a $\mathrm{BBH}$ merger, we aim at providing predictions for this afterglow at several bands of observational interest, with the goal of providing a theoretical framework against which future observations can put to test a range of theoretical models such as the ones discussed above. Our populationsynthesis simulations yield the density distribution expected from a population of field BBHs, and hence this is a robust aspect of the calculation. For the energetics, we adopt as a reference the value required by the Fermi counterpart, since this has been the one mostly used in the literature, and the proposed theory models are generally able to accomodate it $^{4}$.

Using our estimates of afterglow-like EM counterparts, we then perform a first comparison using the available data from the past GW events, and hence predict how many $\mathrm{BBH}$ merger events are needed in order to rule out models predicting an energy release comparable to what required by the Fermi counterpart.

\footnotetext{
${ }^{4}$ However, given the anaytical scalings of the afterglow luminosity with energy, our results can be easily rescaled
}

\subsection{Afterglow model for relativistic outflows}

In the following we adopt the generic afterglow model developed by Sari, Piran \& Narayan (1998) for a relativistic, adiabatic shock. Radiation is expected as a result of synchrotron emission by1 shock-accelerated electrons. We compute such emission within the standard synchrotron model The electrons are assumed to have a power-law distribution of Lorentz factors $\gamma$ above a minimum value $\gamma_{m}$, and are able to loose rapidly energy to radiation if their $\gamma$ is larger than a threshold value $\gamma_{c}$. The resulting spectral energy distribution is then (e.g., Sari, Piran \& Narayan 1998)

$$
F_{\nu}=F_{\nu, \max } \begin{cases}\left(\nu / \nu_{c}\right)^{1 / 3}, & \nu<\nu_{c} \\ \left(\nu / \nu_{c}\right)^{-1 / 2}, & \nu_{c} \leqslant \nu<\nu_{m} \\ \left(\nu_{m} / \nu_{c}\right)^{-1 / 2}\left(\nu / \nu_{m}\right)^{-p / 2}, & \nu \geqslant \nu_{m}\end{cases}
$$

where $\nu_{c} \equiv \nu\left(\gamma_{c}\right)$ and $\nu_{m} \equiv \nu\left(\gamma_{m}\right)$.

On the other hand, if the criterium $\gamma_{c}>\gamma_{m}$ is satisfied, only electrons with $\gamma_{e}>\gamma_{c}$ can cool efficiently. Under this condition, the spectrum takes the functional form (e.g., Sari, Piran \& Narayan 1998)

$$
F_{\nu}=F_{\nu, \max }\left\{\begin{array}{ll}
\left(\nu / \nu_{m}\right)^{1 / 3}, & \nu<\nu_{m} \\
\left(\nu / \nu_{m}\right)^{-(p-1) / 2}, & \nu_{m} \leqslant \nu<\nu_{c} \\
\left(\nu_{c} / \nu_{m}\right)^{-(p-1) / 2}\left(\nu / \nu_{c}\right)^{-p / 2}, & \nu \geqslant \nu_{c}
\end{array} .\right.
$$

In the above equations, the parameter $p$ represents the power-law index of the electron energy distribution. The maximum flux intensity is achieved when $F_{\nu}=F_{\nu, \max }$, where (Sari, Piran \& Narayan 1998)

$$
F_{\nu, \max }=110 n^{1 / 2} \xi_{B}{ }^{1 / 2} E_{52} d_{28}^{-2}(1+z) \mathrm{mJy} .
$$

This formalism assumes that the magnetic field energy density in the shock rest frame is a fraction $\xi_{B}$ of the equipartition value, and the electrons carry a fraction $\xi_{e}$ of the dissipated energy. $E_{52}$ is the explosion energy in units of $10^{52} \mathrm{erg}$, while $d_{28}$ is the luminosity distance in units of $10^{28} \mathrm{~cm}$, and $t_{d}$ is the time in days, as measured in the observer frame, since the beginning of the burst. The relevant frequencies at which the spectral index changes are the cooling frequency $\nu_{c}$ (corresponding to energies at which radiative losses over the shock's lifetime are significant) and the synchrotron frequency $\nu_{m}$ (the minimal energy of the radiating electrons), respectively given by the following expressions (Sari, Piran \& Narayan 1998)

$$
\nu_{c}(t)=2.7 \times 10^{12} n_{1}^{-1} \xi_{B}{ }^{-3 / 2} E_{52}^{-1 / 2} t_{\mathrm{d}}^{-1 / 2}(1+z)^{-1 / 2} \mathrm{~Hz},
$$

and (Sari, Piran \& Narayan 1998)

$$
\nu_{m}(t)=5.7 \times 10^{14} \xi_{e}^{2} \xi_{B}^{1 / 2} E_{52}^{1 / 2} t_{\mathrm{d}}^{-3 / 2}(1+z)^{1 / 2} \mathrm{~Hz}
$$

Additionally, at low frequencies, synchrotron self-absorption can become important. The self-absorption frequency below which the spectrum becomes optically thick is given by (Yost et al. 2003)

$$
\nu_{a}(t)=4.2 \times 10^{8} f(p)\left(\xi_{e}^{*}\right)^{-1} \xi_{B}^{0.2} E_{52}^{0.2} n^{0.6}(1+z)^{-1} \mathrm{~Hz},
$$

where $f(p)=[(p+2)(p-1) /(3 p+2)]^{0.6}$ and $\xi_{e}^{*}=\xi_{e}(p-$ $2) /(p-1)$. Below this frequency, the spectrum declines more rapidly, as $\nu^{2}$. 


\section{RESULTS}

\subsection{Chirp mass, total mass, and merger sites}

The left panel of Fig. 2 shows the distribution of chirp mass, $M_{\text {chirp }}=M_{1}^{3 / 5} M_{2}^{3 / 5}\left(M_{1}+M_{2}\right)^{-1 / 5}$, and of total mass, $M_{\text {tot }}=M_{1}+M_{2}$, for our three representative metallicities in model M10, while the right panel shows the same distributions for $Z=10 \% Z_{\odot}$, and our three representative models (M10/M13/M15). Note that, for relatively low mass GW events, the GW signal in the LIGO sensitive frequency band is dominated by the inspiral phase, and the chirp mass is the most accurately measured parameter (Ajith \& Bose 2009). As expected, the mass distribution has a strong metallicity dependence, reflecting the significant amount of mass lost to winds during the evolution of the more metal rich progenitors stars. On the other hand, the dependence on the specific model at the same metallicity is considerably weaker, with model M10 displaying an enhancement in mass at the higher end of the distribution. This results from the larger amount of fallback for the more massive BHs. The points overlaid on the plot show the mass measurements from the first two observational runs (Abbott et al. 2016a, 2017c; Abbott et al. 2017; Abbott et al. 2017d). A comparison between theory and data suggests that, within the isolated binary formation scenario, the BBHs which merged where likely formed within a range of metallicities. However, since the distributions of merger locations are dependent only very weakly on the progenitor stars metallicity, we can safely adopt a single value of metallicity for the calculations which mainly depend on locations.

In Fig. 3 we show the distribution of projected distances (from the galaxy center) of the merger sites for our three representative models and the three galaxy types, for $Z=0.1 Z_{\odot}$. The orientation angle of the galaxy with respect to the line of sight in each Monte Carlo realization is drawn from a random distribution. All three models predict a sizable fraction of mergers to occur at very large distances, with a fraction $\sim 10-30 \%$ (depending on the model) expected to occur outside of spiral and elliptical galaxies (formal size set at $100 \mathrm{kpc}$ ). As expected, model M13, with the largest natal kicks for the $\mathrm{BHs}$, is the one which predicts the largest fraction of mergers to be located at the largest distances. The fraction of $\mathrm{BBH}$ merger events expected to occur outside of dwarf hosts (formal size set at $10 \mathrm{kpc}$ ) is larger, ranging from $\sim 40 \%$ in the model with the smallest natal kicks to $\sim 60 \%$ in the model with the largest kicks. This larger fraction is expected as a result of the shallower potential of the dwarf galaxy.

As a reference for the observability of a putative EM counterpart, the figure also shows, with vertical lines, the projected distances corresponding to 1 arcsec for a galaxy at the three representative redshifts of $z=0.05,0.1,0.2$, all within the aLIGO horizon, at least for the most massive BHs. This shows that observational facilities with sub-arsec localization capabilities could measure potential counterpart off-sets / positions within the host galaxy.

For the computation of afterglow-like EM radiation accompanying $\mathrm{BBH}$ mergers, the ambient density of the medium at the merger sites plays an important role, since the afterglow peak flux scales as $n^{1 / 2}$ (see Eq. 18). Fig. 4 shows the number density distribution predicted by our models, for evolution in a spiral, elliptical, and dwarf galaxy.

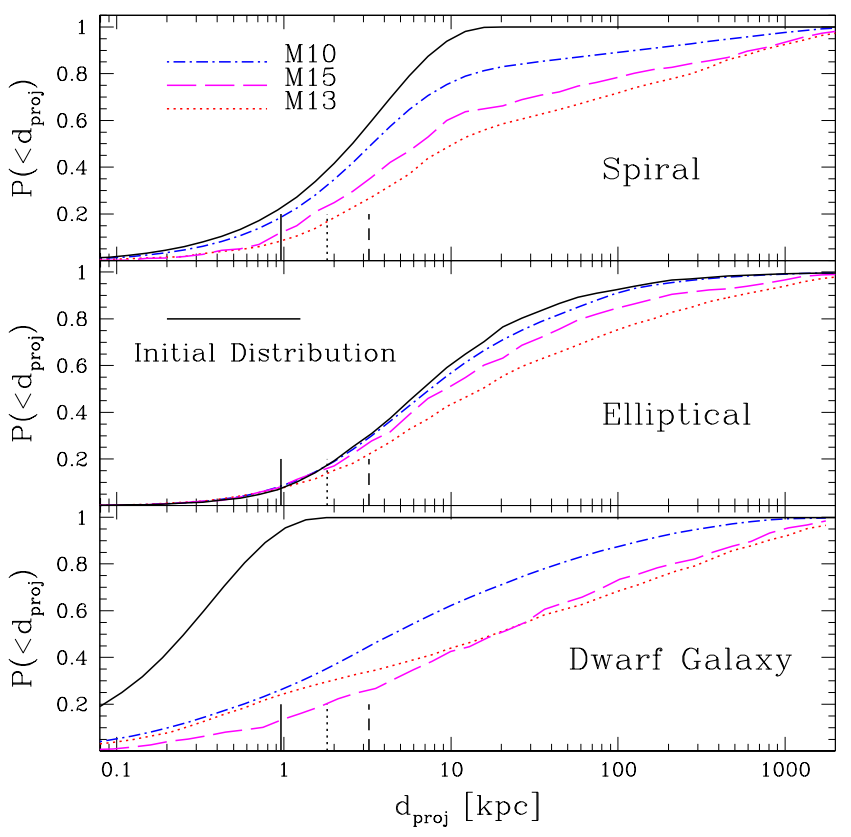

Figure 3. The fraction of $\mathrm{BBH}$ binaries which merge within a projected distance $d_{\text {proj }}$ of their host galaxy center. Distances are the largest in the M13 model, reflecting the larger kicks at birth. A fraction $\sim 10-30 \%$ (with precise value dependent on the model) is expected to be found outside of their elliptical / spiral hosts (formal size set to $100 \mathrm{kpc}$ ). The fraction of $\mathrm{BBH}$ merger events expected to occur outside of dwarf hosts (formal size set to $10 \mathrm{kpc})$ is larger $(\sim 40-60 \%)$ due to the shallower galaxy potential. For each model, the dependence of distances on metallicity is negligible, hence only the $Z=0.1 Z_{\odot}$ case is shown. The three vertical lines correspond to an angular distance of 1 arcsec for a galaxy at a redshift of $z=0.05$ (solid line), $z=0.1$ (dotted line) and $z=0.2$ (dashed line).

Higher ambient densities are generally expected in model M10, given the smaller distances that the binaries have traveled prior to merging. Correspondingly, this scenario will predict brighter afterglows, as quantified in Sec. 5.3.

\subsection{Spin evolution of the primary $\mathrm{BH}$ as a result of accretion during binary evolution}

In the binary evolutionary scenario under consideration, the calculation of the spins that we perform - following the formalism described in Sec. 3.4 - and report on here refers to the evolution of the spin of the primary $\mathrm{BH}$ (i.e. the $\mathrm{BH}$ born first, which is typically the more massive of the two BHs at merger time) as a result of mass accretion from the companion star during evolution. As evident by Eq. (10), the angular momentum (and hence the spin) of the BH following accretion depends on the amount of angular momentum possessed by the accreted mass, as well as on the initial 


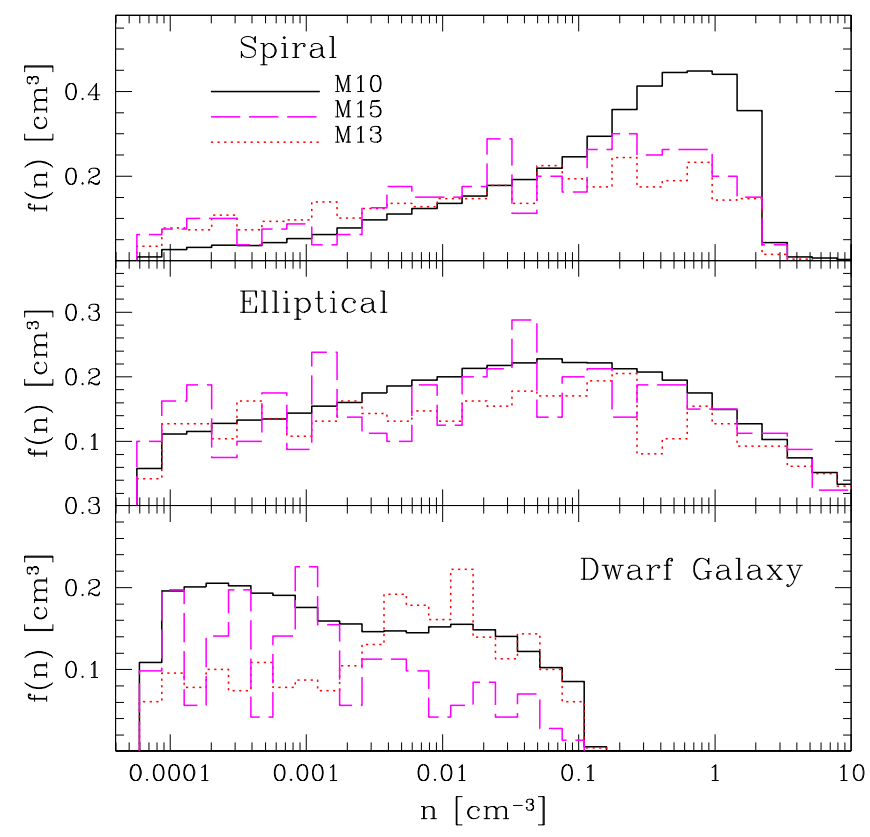

Figure 4. The density distribution at the sites of BBH mergers, for our galaxy potentials and three evolutionary models. Densities tend to be higher in the M10 model, reflecting the smaller natal kicks of the BHs. The densities around the merger sites are important for the possible production of a shock-driven afterglow.

angular momentum possessed by the BH immediately following the supernova (SN) explosion. Since the distribution of $\mathrm{BH}$ spins following the SN explosion is not well known, here we perform calculations for three initial values of the primary $\mathrm{BH}$ spin (namely $a_{\text {in }}=0,0.25,0.5$ ), so that the net effect of the increase due to accretion (which we accurately compute here) can be immediately inferred.

The left panel of Fig. 5 shows the expected spin distribution in model M10 for the three representative metallicities and an inital BH spin equal to zero. In all the cases, the spin gained by the $\mathrm{BHs}$ is rather marginal. The distribution peaks at $a \sim 0.1$, for the $1 \%$ and $10 \%$ solar metallicities, and at $a \sim 0.2$ for the solar metallicity case. The larger spin acquired by the BHs from higher metallicity stars is due to the fact that, while the accreted mass during the common evolution phase is not significantly dependent on metallicity, however, the mass of the BHs which are accreting is typically smaller at higher metallicities, hence resulting in a larger spin.

The middle panel of Fig. 5 shows the final spin distribution for model M10 at $10 \%$ metallicity, for three initial values of the initial spin of the BHs at birth. The general trend is that of a relatively smaller increase for the largest initial spins, as expected. Last, the right panel shows the dependence of the spin distribution on the different evolutionary model assumptions. Compared to model M10, models M13 and M15 display a longer tail of larger spins. This is mainly due to the fact that model M10 produces a relatively higher fraction of more massive BHs compared to models M13 and M15.

So far, LIGO measurements of $\mathrm{BH}$ spin magnitudes have been inconclusive (Abbott et al. 2017c). This originates from the fact that gravitational waveforms are not very sensitive to $\mathrm{BH}$ spins (Abbott et al. 2017c), unless the BH spins are high and precess around (are tilted with respect to) the binary angular momentum. In the four current BBH merger detections (GW150914, LVT151012, GW151226, GW170104) no significant precession was measured, and only in one event (GW151226) a non-zero spin magnitude of at least one $\mathrm{BH}$ is required.

Our results show that accretion in progenitor binaries of massive $\mathrm{BBH}$ mergers is not an efficient process to spin up BHs. For very low natal BH spins $\left(a_{\text {in }} \approx 0\right)$, the final $\mathrm{BH}$ spin in not larger than $0.2-0.3$, and for higher initial $\mathrm{BH}$ spins $\left(a_{\text {in }} \gtrsim 0.5\right)$ the $\mathrm{BH}$ spin remains almost unaffected by accretion. This is a direct result of the fact the accretion onto a $\mathrm{BH}$ in the common envelope phase (the major accretion event for typical $\mathrm{BBH}$ progenitor) is not estimated to occur at rates much higher than a few percent of the Bondi rate (Ricker \& Taam 2008; MacLeod \& Ramirez-Ruiz 2015; Murguia-Berthier et al. 2017). In other words, our results show that LIGO will be measuring the natal BH spin in $\mathrm{BBH}$ mergers if they originate from the classical formation scenario discussed here (Belczynski et al. 2016b). This is of fundamental importance, since $\mathrm{BH}$ natal spins can be potentially used to infer information about the angular momentum/rotation of the massive stars progenitors of these BHs. Rotation and angular momentum transport in massive stars are basic missing blocks of stellar evolution theory.

If, for example, LIGO BHs are found to typically have very low spins, it will indicate that angular momentum is lost rather effectively from massive stars, and the TaylerSpruit dynamo (Spruit 1999) may be a process that operates in massive stars. However, that would also indicate that LIGO BHs originate from a different population than Galactic and extra-galactic High-Mass X-ray Binaries, which all show very high spins $(a \gtrsim 0.9$; see Tab. 1 in Fragos \& McClintock 2015). If, on the other hand, BH spins were to be found to be generally high, it would mean that angular momentum transport is a rather inefficient process (i.e. the core must decouple from the envelope early on in the massive star evolution). In this last case, a variety of long GRB central engines based on single massive star progenitors (e.g., MacFadyen \& Woosley 1999), could also be realized in BBH mergers. Last, if a mixed population (low and high spins) of LIGO BHs is found by future detections, it will point to mild coupling of layers within a massive rotating star, moderated by the effects of wind mass loss that depends on star mass and metallicity (e.g., Geneva stellar models; Eggenberger, Montalbán \& Miglio 2012; Georgy et al. 2013). Such a model would naturally explain high spins of low mass BHs (like the ones observed in HMXB in very local Universe), along with low spin $\mathrm{BHs}$ that have high masses $\left(M_{\mathrm{BH}} \gtrsim 20-30 \mathrm{M}_{\odot}\right.$; Belczynski et al. 2017). 

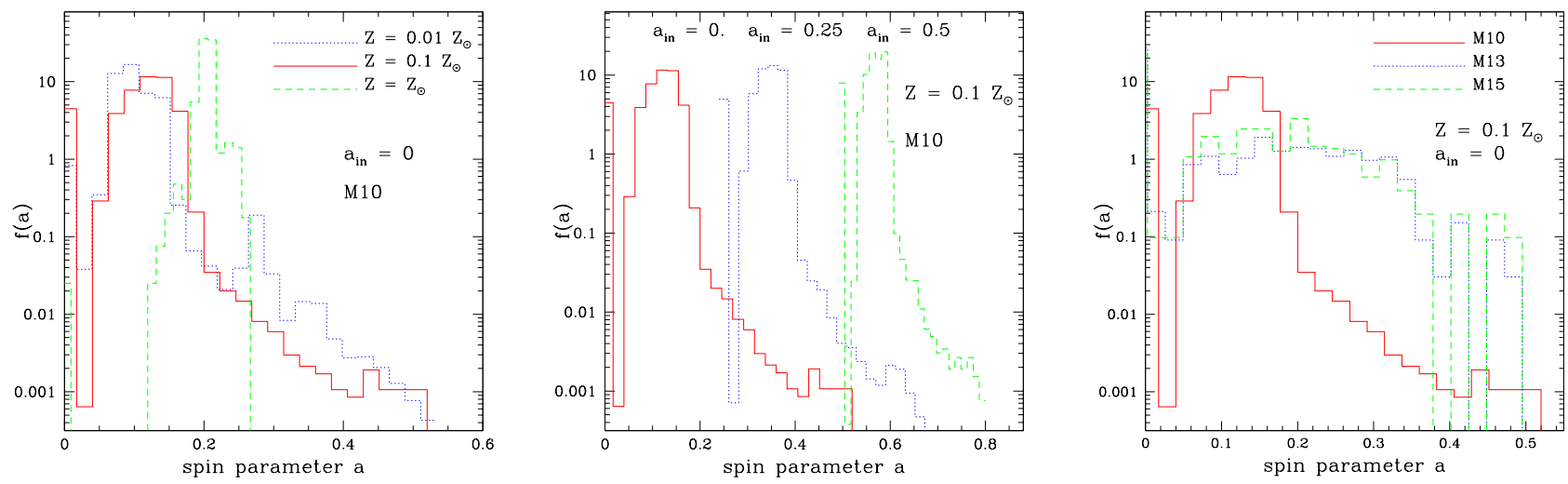

Figure 5. The distribution of the spin parameter of the primary BH due to accretion of matter from the companion during the binary evolution. Left: Dependence on metallicity for the M10 model, assuming that the initial spin of the BH (following the SN explosion) is zero. Middle: Dependence of the spin distribution on the initial BH spin, for the M10 model at 10\% of solar metallicity. Right: The spin distribution in the three evolutionary models studied here, at $10 \%$ metallicity and assuming zero initial spin.

\subsection{EM counterparts to BBH mergers}

\subsubsection{Predictions for energetics comparable to that of the Fermi counterpart}

In the following, we use the radiation model described in Sec. 4, coupled with the density distribution at the $\mathrm{BBH}$ merger sites calculated in Sec. 5.1, to estimate the expected brightness distribution of afterglows possibly associated with $\mathrm{BBH}$ merger events.

We compute the predicted afterglow luminosities under the assumption that each $\mathrm{BBH}$ merger event is associated with an explosive release of energy. For simplicity, and for the lack of other reference numbers, we take as a representative case the energetics of the candidate $\gamma$-ray counterpart to GW150914 reported by the Fermi collaboration (Connaughton et al. 2016), i.e. $E=10^{49} \mathrm{erg}$. We remind the reader (cfr. Sec.4.1) that this implies that the high energy $\gamma$-ray emission must be produced within a jet of angular size $\lesssim 50^{\circ}$ and be relativistic beamed. We adopt $\xi_{B}=0.01$ and $\xi_{e}=0.1$, as typical values inferred from broad-band afterglow modeling in the 'standard' GRBs (Wijers \& Galama 1999; Panaitescu \& Kumar 2001). Using a single value for $E, \xi_{B}, \xi_{e}$ (rather than assuming some arbitrary distributions) allows us to (a) highlight the dependence on the different evolutionary models (which predict different merger sites and hence medium densities), and (b) present results that can be immediately rescaled to other values of these parameters (which may be motivated by future observations), thanks to the analytical dependence of the afterglow flux on these parameters (see Sec.4).

Fig. 6 shows the distribution of afterglow luminosities in the $2-10 \mathrm{keV}$ band for our three representative models and three galaxy types. Since, as discussed above, the merger sites have very little dependence on metallicity, we only show results for $Z=10 \% Z_{\odot}$. In addition to showing predictions at an observing time of $1 \mathrm{hr}$ after the merger, as would be typical following a $\gamma$-ray localization, we also display, for the reference M10 model, the expected flux distribution at 1 day after the merger, to reflect what realized with the
X-ray follow ups to the LIGO-detected events in runs O1 and O2. The vertical lines indicate the minimum luminosity that would be detectable by Swift/XRT in a follow-up search with sensitivity similar to the one achieved for GW150914 $\left(F_{\text {lim }} \approx 2.5 \times 10^{-13} \mathrm{erg} \mathrm{s}^{-2} \mathrm{~cm}^{-2}\right.$ in the $2-10 \mathrm{keV}$ band; see Abbott et al. 2016c) for a burst located at the three representative redshifts of $z=0.05,0.1,0.2$ (all within the LIGO $\mathrm{BBH}$ horizon). The probability of detecting an X-ray counterpart is in the range of $65-85 \%$ depending on the evolutionary model and galaxy type for the closest elliptical/spiral galaxy, and between 40-70\% for the farthest galaxy. A larger fraction of brighter events is expected in the M10 model, due to the generally smaller distances (and hence higher densities) of the merger sites from the galaxy centers. Note that the probabilities flatten out at low luminosities with values $<1$ as a result of fact that a fraction of $\mathrm{BBH}$ mergers is predicted to occur outside of the host galaxy, in the intergalactic medium. The luminosities of those events (which would bring the total cumulative probabilities to 1) are lower than the lowest limit in the figure. In the case of dwarf hosts, for the same emission parameters $\left(E, \xi_{e}, \xi_{B}\right)$ used for the massive galaxies, the luminosities are lower, due to the smaller number densities. Additionally, a larger fraction of events is expected to be 'naked', due to the mergers occurring outside the host. This fraction can be directly read from the flattening of the probability distributions at low luminosities: up to a fraction $\sim 30-50 \%$ (depending on the magnitude of the natal kicks and the redshift of the host) could have a nonnegligible afterglow-like EM counterpart.

Fig. 7 shows the same distributions, but in the optical after 1 day. The vertical lines, corresponding to the minimum detectable luminosity for events at the same representative redshifts as in Fig. 6, have been computed assuming a $5 \sigma$ limiting magnitude of $r \approx 22.4 \mathrm{mag}$ ( $\mathrm{AB}$ system, equivalent to a limiting flux of $F_{\text {lim }} \approx 4 \mu \mathrm{Jy}$ ), comparable to the most sensitive optical searches carried out in this band during the follow-up of GW150914 (see Table 1 in Abbott et al. 2016c). An inspection of Fig. 7 shows that detection in the optical is not likely for a merger in a dwarf galaxy, even 


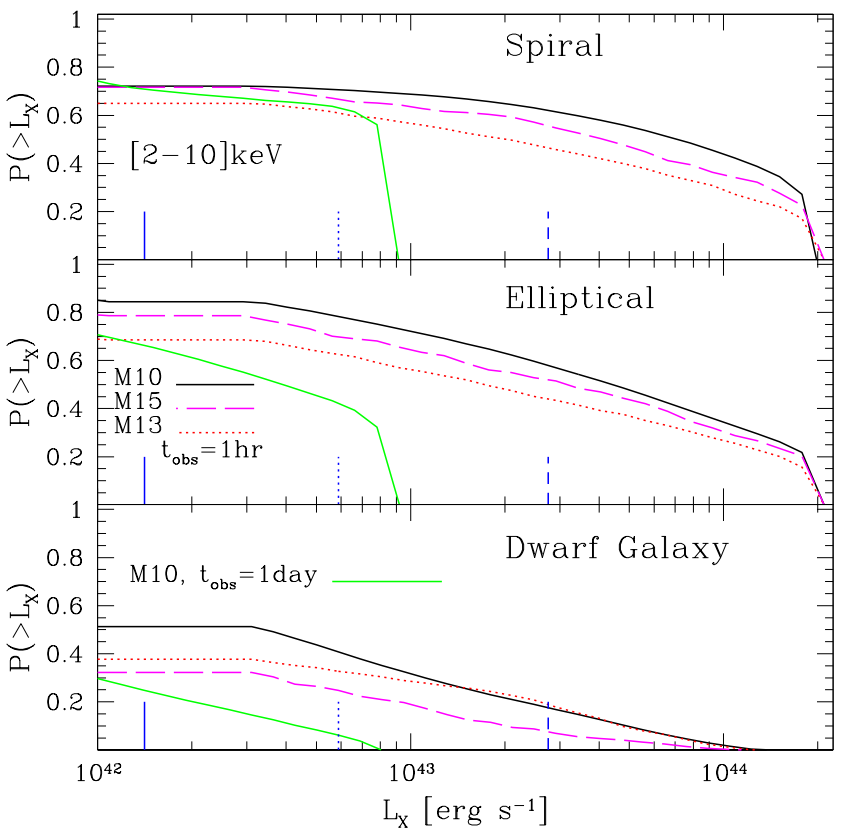

Figure 6. The cumulative probability distribution of afterglow luminosities in the $2-10 \mathrm{keV}$ band at $1 \mathrm{hr}$ after the burst for our three representative models, and at 1 day for model M10, as a reference for comparison to past observations. The vertical lines indicate the minimum luminosity that would be detectable by Swift/XRT (unabsorbed $2-10 \mathrm{keV}$ flux of $F_{\text {lim }} \approx$ $2.5 \times 10^{-13} \mathrm{erg} \mathrm{s}^{-2} \mathrm{~cm}^{-2}$, see text) for a burst located at $z=0.05$ (solid line), $z=0.1$ (dotted line), $z=0.2$ (dashed line), respectively. Note that the probabilities do not saturate at 1 , reflecting the fraction of much dimmer afterglows resulting from mergers outside of the host galaxy, in the intergalactic medium. This probability would need to be further reduced by the beaming factor if the emission is produced within a collimated jet. In particular, since the X-ray luminosity is expected to be produced when the flow is still relativistic, those probabilities should be reduced by a factor of $\sim 3$, with the beaming factor constrained by the $\gamma$-ray limits on all the BBH GW merger events detected so far (cfr. $\S 4.1)$.

at a low redshift of $z \sim 0.05$. For massive galaxies, the detection probability becomes more sizable, but only at low redshifts. In particular, for $z=0.05$, a fraction between 30$60 \%$ of events could be detected in the optical. This fraction becomes $10 \%$ or smaller already at $z=0.1$.

Fig. 8 shows the probability distributions in the radio band, at $1.4 \mathrm{GHz}$ after 1 week from the explosive event. Here, the reference threshold flux corresponding to the minimum detectable luminosities at the redshifts of $0.05,0.1,0.2$ is set at the $5 \sigma$ threshold flux for the Karl G. Jansky VLA (A configuration) for $1 \mathrm{hr}$ integration on-source $\left(F_{\text {lim }} \approx 50 \mu \mathrm{Jy}\right)$. For mergers at a redshift of 0.2 , corresponding to the highest redshift LIGO BBH merger so far (Abbott et al. 2017), about $25-45 \%$ of events could be detected as radio after-

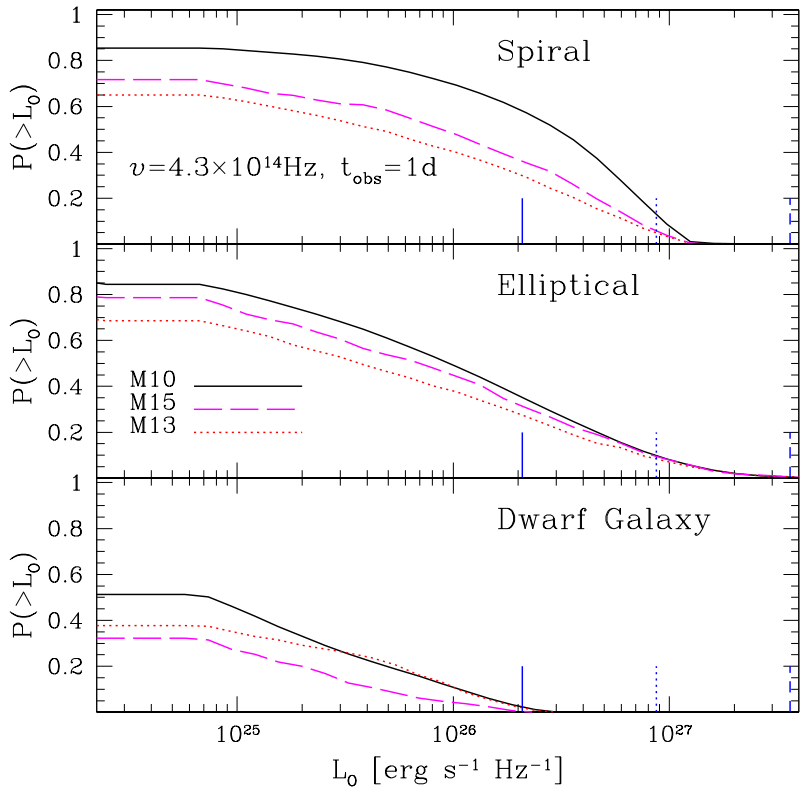

Figure 7. Same as in Fig.6, but at the frequency of $\nu=4.3 \times$ $10^{14} \mathrm{~Hz}$ (falling in the $\mathrm{R}$ band) at 1 day after the burst. A limiting flux of $F_{\text {lim }} \approx 4 \mu \mathrm{Jy}$ (see text) has been assumed to set the values of the minimum luminosity for detection at a redshift of 0.05 (solid line), 0.1 (dotted line), 0.2 (dashed line). Some reduction in the probability due to beaming should be accounted for also in the optical, albeit at a lower level than in the $\mathrm{X}$ and $\gamma$-rays.

glows, depending on the model and galaxy type, for massive galaxies, while for dwarf galaxies the detection probability is non-negligile only at lower redshifts, being about $20 \%$ for events at $z=0.1$. Note that, for the particular choice of afterglow parameters adopted here (namely $E, \xi_{e}, \xi_{B}$ ), and the range of densities probed by the merger events, the afterglow spectrum is in the regime described by the middle branch of Eq. (18). In this case, the flux at the same observation time is higher at lower frequencies. However, given the sensitive parameter dependences of Eq. (19) and Eq. (20), the frequency-dependence across the bands can be reversed for other combinations of the relevant parameters.

We finally note that, up to this point, our calculations have estimated the brightness of possible afterglow counterparts independently of their visibility due to beaming effects. However, an afterglow-like EM counterpart to a GW event may come from a relativistic jet of size $\theta_{\text {jet }}<\pi / 2$, and the discussion of $\S 4.1$ has shown that, for an emission model with energetics comparable to those inferred for GW150914, the high energy emission must be confined within a jet of $\lesssim 50^{\circ}$. This constraint, which was derived for the $\gamma$-ray emission from the Fermi follow ups during the O1/O2 runs, likely extends also to the X-rays, which are produced when the shock is still relativistic (and more moderately to the optical). Therefore our computed probabilities for the observability of afterglow-like emission should be reduced by the beaming factor $\left(1-\cos \theta_{\text {jet }}\right) \sim 1 / 3$ in the $\mathrm{X}$-rays if interpreted as detection probability of an EM counterpart fol- 


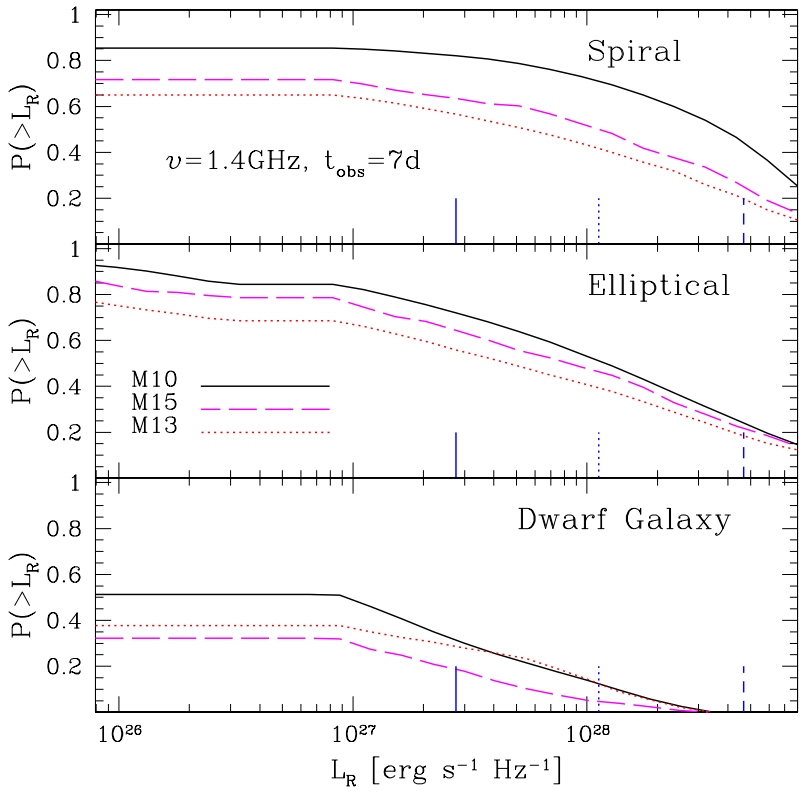

Figure 8. Same as in Figs. 6 and 7 but in the radio $(1.4 \mathrm{GHz})$ at 7 days after the burst. A limiting flux of $F_{\text {lim }}=50 \mu \mathrm{Jy}$ (see text) has been assumed to set the values of the minimum luminosity for detection at a redshift of 0.05 (solid line), 0.1 (dotted line), 0.2 (dashed line). In the radio band, the emission is expected to be close to isotropic, and hence the computed probabilities are not expected to be affected by beaming effects.

lowing a GW event independently of a $\gamma$-ray detection. A milder reduction is expected in the optical, while the radio emission should be close to isotropic.

\subsubsection{Model constraints from past observations}

Among the LIGO BBH events, GW150914 was probably the one that enjoyed the most extensive and coordinated EM follow-up observations (Abbott et al. 2016c), and the one for which a possible association with a gamma-ray transient was also claimed (Connaughton et al. 2016). Here we make the assumption that the EM follow-up effort that accompanied GW150914 is representative of the typical effort that will be put in place by the community in the future hunt for EM counterparts to BBH mergers. In this framework, it is thus reasonable to assume that, as for the case of GW150914, optical surveys will be able to cover typical localization areas of order $\sim 100 \mathrm{deg}^{2}$ down to $r<22.4 \mathrm{mag}$ within a timescale of order $\sim 1 \mathrm{~d}$ since the merger (Abbott et al. 2016c). X-ray observatories such as e.g. Swift may instead cover localization areas of $\lesssim 5 \mathrm{deg}^{2}$ within a timescale of $\sim 1$ day since the merger and down to a limiting $2-10 \mathrm{keV}$ unabsorbed flux of $2.5 \times 10^{-13} \mathrm{erg} \mathrm{cm}^{-2} \mathrm{~s}^{-1}$ (Abbott et al. 2016c).

For what concerns typical GW localization areas, for massive BBHs located at $z \lesssim 0.1$, and as demonstrated in the case of GW170814 (Abbott et al. 2017) we can expect the two LIGO and Virgo detectors to provide $90 \%$ credible areas of $\sim 60 \mathrm{deg}^{2}$. Thus, while optical follow-up observations will be able to cover basically all of of the GW localization areas, deep X-ray follow-up observations may only end up covering $\lesssim 10 \%$ of the $\mathrm{GW}$ localization area.

Based on the above considerations and on our estimates, optical follow-ups of massive BBHs at $z \lesssim 0.1$ will be limited by the theoretical expectations that only $\approx 5-10 \%$ of the $\mathrm{BBH}$ mergers located in spiral/elliptical galaxies are expected to have an optically detectable $(r<22.4 \mathrm{mag})$ afterglow. Thus, in order to exclude the predictions presented in this work at the $\gtrsim 90 \%$ confidence, we would need to collect a sample of $\sim 25-50 \mathrm{BBH}$ events i.e. with energetics comparable GW150914 with deep optical follow-up observations of their GW localization areas. Our estimates also show that a fraction $\approx 40-70 \%$ of the $\mathrm{BBH}$ mergers in spiral/elliptical galaxies at $z \lesssim 0.1$ would have an X-ray afterglow detectable at $1 \mathrm{~d}$ since merger. But, for these we expect only $\sim 10 \%$ of the localization area to be covered with X-ray follow-up observations. Thus, overall, we would need a sample of $\sim 30-60$ GW150914-like BBH events with deep $\mathrm{X}$-ray follow-up observations to exclude the predictions presented here at the $90 \%$ confidence level. Applying the same constraints on beaming as derived from the $\gamma$-rays, the number of $\mathrm{BBH}$ events quoted above would need to be multiplied by a factor of about 3 .

In summary, because it appears likely that a sample $\sim 50-100 \mathrm{BBH}$ events will be collected by the advanced detectors in their upcoming observing runs (Abbott et al. $2016 \mathrm{~g}$ ), testing the predictions presented here is in fact within reach, as long as optical/X-ray follow-up efforts continue to be pursued by the community. The above considerations also demonstrate that so far the lack of detections of radio/optical/X-ray afterglows from the 4 significant LIGO/Virgo BBH detections (e.g. Copperwheat et al. 2016; Evans et al. 2016; Kasliwal et al. 2016; Morokuma et al. 2016; Palliyaguru et al. 2016; Smartt et al. 2016b,a; Bhalerao et al. 2017; Corsi et al. 2017; Kawai et al. 2017) does not rule out the hypothesis that some or all of GW150914-like events may be accompanied by gamma-ray transients giving rise to multi-band afterglows.

Finally, we note that while our estimates for the detectability of radio afterglows with sensitive $\mathrm{GHz}$ telescopes such as the VLA are promising ( $\gtrsim 30 \%$ for BBHs located in spiral/ellipticals at $z \lesssim 0.1$ ), these telescopes also have small field of views and thus the EM follow-up at these wavelengths will likely rely on accurate localizations provided by the identification of optical or X-ray counterparts.

\section{SUMMARY}

In light of the detection of GWs from BBH mergers, and the massive efforts at searching for EM counterparts from such events, here we have performed a study aimed at predicting the statistical properties of the merged $\mathrm{BBH}$ population within their host galaxies, for $\mathrm{BHs}$ produced via massive stars evolution in isolated binaries. In addition to calculating the mass function (provided in terms of both chirp and total mass), for three values of the metallicity, we have computed the spin distribution of the primary $\mathrm{BH}$ as a result of accretion of mass from the secondary during the close binary evolution. Of particular importance for optimizing follow-up 
strategies, we provided the expected distance distribution within the galaxy potential, for a massive spiral, a massive elliptical, and a dwarf galaxy (modeled as a scaled-down version of a spiral). We considered the two most extreme models (as well as one in between) for the kicks received by the BHs at birth. As such, our results span the expected range for $\mathrm{BBH}$ mergers within the isolated binary scenario. The distribution of the merger sites further allows us to compute (for a given galaxy model) the ISM density distribution for the merger locations.

Last, we entertained the possibility that BBHs may be accompanied by the formation of an outflow and a release of electromagnetic energy via a shock. This was motivated by the tentative detection of a $\gamma$-ray counterpart to GW150917, and a number of ideas and models aimed at explaining such emission (see $\S 1$ and $\S 4.1$ for references and details). However, the lack of detection of $\gamma$-ray counterparts to the other $5 \mathrm{BBH}$ GW events (including the LIGO/Virgo trigger) implies that either the $\gamma$-ray luminosities are lower, or that the emission is beamed within an angle $\lesssim 50^{\circ}$. By adopting (for simplicity) the $\gamma$-ray luminosity inferred for the GW150917 candidate counterpart, we have hence made the implicit assumption that the $\gamma$-ray emission is beamed to within $\lesssim 50^{\circ}$. With this caveat in mind, and the computed distributions of ISM densities at the merger locations, we could then calculate the broad-band afterglow luminosities expected from these events. Our estimates are particularly instructive since, especially at the longer (radio) wavelength, beaming is less important and hence observational limits can help set tighter constraints on proposed emission models.

Our results can be summarized as follows:

(i) The mass function has a strong metallicity dependence, as expected. A chirp mass $\sim 20 M_{\odot}$ requires metallicities $\lesssim 0.1 Z_{\odot}$. The influence of natal kicks is much weaker than the influence of metallicity on the $\mathrm{BBH}$ merger mass function.

(ii) The accreted mass from the progenitor star of the secondary $\mathrm{BH}$ to the primary $\mathrm{BH}$ during the period of mass transfer increases the BH spin by an amount which, in the standard model M10 is about 0.1 for $0.01 \%$ and $0.1 \% Z_{\odot}$, and about 0.2 for $Z_{\odot}$. BHs formed at high metallicity are less massive, and hence easier to spin up for the same amount of accreted mass. Additionally, the spin increase, for the same amount of accreted mass, is slightly smaller for larger initial natal spins of the primary BH (see also Belczynski et al. 2017). For the same initial conditions, models M13 and M15 produce a generally larger fraction of high spin BHs, with a flat spin distribution up to $a \sim 0.35$ if the initial spin of the primary $\mathrm{BH}$ is zero.

(iii) The distribution of merger sites (and hence of projected distances/angular offsets) within the host galaxies spans a large range of values, with a fraction $\sim 10-30 \%$ for massive galaxies (with precise value depending on the model and galaxy type) occurring outside of the galaxy, at distances $R \gtrsim 100 \mathrm{kpc}$. This fraction of mergers outside of the hosts is larger $(\sim 40-60 \%)$ for a dwarf galaxy, here modeled as a spiral with mass of $0.1 \%$ that of the Milky Way. As expected, in the model with the largest natal kicks (M13), there is a larger fraction of mergers occurring outside of the galaxy, and more generally at larger distances from the center. These offsets could be easily measured by any ob- servational facility with sub-arcsec localization capabilities, if the merger is associated with EM emission.

(iv) Using the distribution of interstellar medium densities derived from the distribution of merger sites within the host galaxies, and assuming that $\mathrm{BBH}$ merger events produce GRB-like counterparts similar to the Fermi candidate counterpart to GW150914, we have computed the resulting afterglow distributions in the X-rays, optical, and radio band. We find that, for massive galaxies, a fraction on the order of $10-30 \%$ of the mergers (where the precise value depends on the assumed natal kick distribution and galaxy type) is expected to be naked, i.e. lacking a bright afterglow at any wavelength. The same fraction is higher, $\sim 40-60 \%$, in dwarf galaxies. These events correspond to mergers that occur outside of the host galaxy, within the diffuse intergalactic medium.

A fraction of the order of $\sim 40-70 \%$ (depending on the model and galaxy type and before beaming corrections) of the mergers in massive galaxies would be accompanied by broad-band afterglow-like emission detectable in X-rays if occurring within the LIGO horizon and if associated with a $\gamma$-ray signal similar to the Fermi candidate counterpart to GW150914 (which would require a kinetic energy on the order of $10^{49}$ ergs). A smaller fraction could also be detectable at radio and optical wavelengths with sensitive enough follow-up instruments. Given the analytical scaling of afterglow flux with energy, our results can be easily scaled should future observations indicate positive detection but point to different values of the energetics.

Our goal for these calculations has been that of providing a theoretical framework against which to test ideas for impulsive energy production accompanying BBH mergers, given the expectation that a sudden energy release would be accompanied by an afterglow-type radiation, in analogy to what observed in connection with the binary neutron star merger event (Abbott et al. 2017). To this purpose, we specifically convolved our theoretical predictions with the observational set up (i.e. time of the observations, sensitivity, sky coverage) of past EM follow ups to LIGO detections of BBH mergers, and assuming this to be typical of future observational campaigns, we provided an estimate of the number of $\mathrm{BBH}$ mergers that needs to be followed in order to rule out explosive-like events accompanying $\mathrm{BBH}$ mergers. In particular, we found that we would need to collect a sample of $\sim 25-50$ GW150914-like BBH events with deep optical follow-up observations of their GW localization areas, and $\sim 30-60$ GW150914-like BBH events with deep X-ray follow- up observations, to exclude the predictions presented here at the $90 \%$ confidence level.

Acknowledgments. We thank an anonymous referee for very useful comments and suggestions, and Valerie Connaughton for providing us with the details of the Fermi GBM follow ups to the $\mathrm{BBH}$ merger events in runs O1 and O2. RP was partly supported by NSF award AST1616157. KB acknowledges support from the Polish National Science Center (NCN) grant: Sonata Bis 2 (DEC2012/07/E/ST9/01360). AC acknowledges support from the NSF CAREER award \#1455090 and also partial support from the Swift Cycle 12 GI program (Grant No. NNX17AF93G). 


\section{REFERENCES}

Abbott B. P. et al., 2016a, Physical Review X, 6, 041015

—, 2016b, Physical Review Letters, 116, 241103

—, 2016c, ApJ, 826, L13

—, 2016d, Physical Review Letters, 116, 061102

—, 2016e, Living Reviews in Relativity, 19, 1

—, 2016f, ApJ, 833, L1

-, 2016g, ApJ, 833, L1

—, 2017, Phys. Rev. Lett., 118, 221101

Abbott B. P. et al., 2017, Phys. Rev. Lett., 119, 141101

Abbott B. P. et al., 2017a, Nature, 551, 85

—, 2017b, ApJ, 848, L13

—, 2017c, Physical Review Letters, 118, 221101

—, 2017d, ApJ, 851, L35

Ajith P., Bose S., 2009, PRD, 79, 084032

Antonini F., Chatterjee S., Rodriguez C. L., Morscher M., Pattabiraman B., Kalogera V., Rasio F. A., 2016, ApJ, 816,65

Bagoly Z., Szécsi D., Balázs L. G., Csabai I., Horváth I., Dobos L., Lichtenberger J., Tóth L. V., 2016, A\&A, 593, L10

Bahcall N. A., Ostriker J. P., Perlmutter S., Steinhardt P. J., 1999, Science, 284, 1481

Bartos I., Kocsis B., Haiman Z., Márka S., 2017, ApJ, 835, 165

Belczynski K., Bulik T., Fryer C. L., Ruiter A., Valsecchi

F., Vink J. S., Hurley J. R., 2010, ApJ, 714, 1217

Belczynski K. et al., 2016a, A\&A, 594, A97

Belczynski K., Holz D. E., Bulik T., O'Shaughnessy R., 2016b, Nature, 534, 512

Belczynski K., Kalogera V., Bulik T., 2002, ApJ, 572, 407 Belczynski K., Kalogera V., Rasio F. A., Taam R. E., Zezas A., Bulik T., Maccarone T. J., Ivanova N., 2008, ApJS, 174,223

Belczynski K. et al., 2017, ArXiv e-prints

Belczynski K., Perna R., Bulik T., Kalogera V., Ivanova N., Lamb D. Q., 2006, ApJ, 648, 1110

Belczynski K., Repetto S., Holz D. E., O'Shaughnessy R., Bulik T., Berti E., Fryer C., Dominik M., 2016c, ApJ, 819, 108

Bhalerao V. et al., 2017, ArXiv e-prints

Bhat P. N. et al., 2016, VizieR Online Data Catalog, 222

Blaauw A., 1961, Bull. of the Astr. Inst. of the Netherlands, 15,265

Bondi H., 1952, MNRAS, 112, 195

Brown G. E., Lee C.-H., Wijers R. A. M. J., Bethe H. A., 2000, Physics Reports, 333, 471

Cavallo G., Rees M. J., 1978, MNRAS, 183, 359

Ciolfi R., Kastaun W., Giacomazzo B., Endrizzi A., Siegel

D. M., Perna R., 2017, PRD, 95, 063016

Connaughton V. et al., 2018, ArXiv e-prints

-, 2016, ApJ, 826, L6

Copperwheat C. M. et al., 2016, MNRAS, 462, 3528

Corsi A. et al., 2017, ArXiv e-prints

Cowperthwaite P. S. et al., 2016, ApJ, 826, L29

Dai L., McKinney J. C., Miller M. C., 2017, MNRAS, 470, L92

de Mink S. E., Cantiello M., Langer N., Pols O. R., Brott

I., Yoon S.-C., 2009, A\&A, 497, 243

de Mink S. E., King A., 2017, ApJ, 839, L7

de Mink S. E., Mandel I., 2016, MNRAS, 460, 3545
Dominik M., Belczynski K., Fryer C., Holz D. E., Berti E., Bulik T., Mandel I., O'Shaughnessy R., 2012, ApJ, 759, 52

Downing J. M. B., Benacquista M. J., Giersz M., Spurzem R., 2010, MNRAS, 407, 1946

—, 2011, MNRAS, 416, 133

Duchêne G., Kraus A., 2013, ARAA, 51, 269

Eggenberger P., Montalbán J., Miglio A., 2012, A\&A, 544, L4

Evans P. A. et al., 2016, MNRAS, 462, 1591

Fong W., Berger E., Margutti R., Zauderer B. A., 2015, ApJ, 815, 102

Fragos T., McClintock J. E., 2015, ApJ, 800, 17

Fraschetti F., 2016, ArXiv e-prints

Fryer C. L., Belczynski K., Wiktorowicz G., Dominik M., Kalogera V., Holz D. E., 2012, ApJ, 749, 91

Georgy C., Ekström S., Granada A., Meynet G., Mowlavi N., Eggenberger P., Maeder A., 2013, A\&A, 553, A24

Greiner J., Burgess J. M., Savchenko V., Yu H.-F., 2016, ApJ, 827, L38

Hernquist L., 1990, ApJ, 356, 359

Hobbs G., Lorimer D. R., Lyne A. G., Kramer M., 2005, MNRAS, 360, 974

Ioka K., Matsumoto T., Teraki Y., Kashiyama K., Murase K., 2017, MNRAS, 470, 3332

Janiuk A., Bejger M., Charzyński S., Sukova P., 2017, New Astr., 51, 7

Kalogera V., Belczynski K., Kim C., O'Shaughnessy R., Willems B., 2007, Physics Reports, 442, 75

Kasliwal M. M. et al., 2016, ApJ, 824, L24

Kawai N., Negoro H., Serino M., Mihara T., Tanaka K., Masumitsu T., Nakahira S., 2017, ArXiv e-prints

Kawamura T., Giacomazzo B., Kastaun W., Ciolfi R., Endrizzi A., Baiotti L., Perna R., 2016, PRD, 94, 064012

Khan A., Paschalidis V., Ruiz M., Shapiro S. L., 2018, ArXiv e-prints

Kimura S. S., Murase K., Mészáros P., 2017, ArXiv e-prints

Kroupa P., Tout C. A., Gilmore G., 1993, MNRAS, 262, 545

Lazzati D., López-Cámara D., Cantiello M., Morsony B. J.,

Perna R., Workman J. C., 2017, ApJ, 848, L6

Li X., Zhang F.-W., Yuan Q., Jin Z.-P., Fan Y.-Z., Liu S.-M., Wei D.-M., 2016, ApJ, 827, L16

Liebling S. L., Palenzuela C., 2016, PRD, 94, 064046

Loeb A., 2016, ApJ, 819, L21

Lyutikov M., 2016, ArXiv e-prints

MacFadyen A. I., Woosley S. E., 1999, ApJ, 524, 262

MacLeod M., Antoni A., Murguia-Berthier A., Macias P., Ramirez-Ruiz E., 2017, ApJ, 838, 56

MacLeod M., Ramirez-Ruiz E., 2015, ApJ, 798, L19

Mandel I., de Mink S. E., 2016, MNRAS, 458, 2634

Marchant P., Langer N., Podsiadlowski P., Tauris T. M., Moriya T. J., 2016, A\&A, 588, A50

Miyamoto M., Nagai R., 1975, PASJ, 27, 533

Morokuma T. et al., 2016, PASJ, 68, L9

Murase K., Kashiyama K., Mészáros P., Shoemaker I., Senno N., 2016, ApJ, 822, L9

Murguia-Berthier A. et al., 2017, ApJ, 835, L34

O'Shaughnessy R., Bellovary J. M., Brooks A., Shen S., Governato F., Christensen C. R., 2017, MNRAS, 464, 2831

O’Shaughnessy R., Kalogera V., Belczynski K., 2005, ApJ, 
620,385

O’Shaughnessy R., Kaplan J., Kalogera V., Belczynski K., 2005, ApJ, 632, 1035

Paczynski B., 1990, ApJ, 348, 485

Palliyaguru N. T. et al., 2016, ApJ, 829, L28

Panaitescu A., Kumar P., 2001, ApJ, 560, L49

Pankow C., Sampson L., Perri L., Chase E., Coughlin S.,

Zevin M., Kalogera V., 2017, ApJ, 834, 154

Paxton B. et al., 2013, ApJS, 208, 4

Perna R., Belczynski K., 2002, ApJ, 570, 252

Perna R., Duffell P., Cantiello M., MacFadyen A. I., 2014, ApJ, 781, 119

Perna R., Lazzati D., Giacomazzo B., 2016, ApJ, 821, L18

Postnov K. A., Yungelson L. R., 2014, Living Reviews in Relativity, 17, 3

Racusin J. L. et al., 2017, ApJ, 835, 82

Rezzolla L., Giacomazzo B., Baiotti L., Granot J., Kouveliotou C., Aloy M. A., 2011, ApJ, 732, L6

Ricker P. M., Taam R. E., 2008, ApJ, 672, L41

Rodriguez C. L., Amaro-Seoane P., Chatterjee S., Rasio F. A., 2017, ArXiv e-prints

Rodriguez C. L., Chatterjee S., Rasio F. A., 2016, PRD, 93, 084029

Ruiz M., Lang R. N., Paschalidis V., Shapiro S. L., 2016, ApJ, 824, L6

Sana H. et al., 2012, Science, 337, 444

Sari R., Piran T., Narayan R., 1998, ApJ, 497, L17

Savchenko V. et al., 2017, ArXiv e-prints

—, 2016, ApJ, 820, L36

Schutz B. F., 1986, Nature, 323, 310

Shakura N. I., Sunyaev R. A., 1973, A\&A, 24, 337

Sigurdsson S., Hernquist L., 1993, Nature, 364, 423

Smartt S. J. et al., 2016a, MNRAS, 462, 4094

—, 2016b, ApJ, 827, L40

Spruit H. C., 1999, A\&A, 349, 189

Stone N. C., Metzger B. D., Haiman Z., 2017, MNRAS, 464, 946

Thorne K. S., 1974, ApJ, 191, 507

Tutukov A. V., Yungelson L. R., 1993, MNRAS, 260, 675

Verrecchia F. et al., 2017, ArXiv e-prints

Villante F. L., Serenelli A. M., Delahaye F., Pinsonneault M. H., 2014, ApJ, 787, 13

Wijers R. A. M. J., Galama T. J., 1999, ApJ, 523, 177

Woosley S. E., 2016, ApJ, 824, L10

Yamazaki R., Asano K., Ohira Y., 2016, Progress of Theoretical and Experimental Physics, 2016, 051E01

Yost S. A., Harrison F. A., Sari R., Frail D. A., 2003, ApJ, 597,459

Zhang B., 2016, ApJ, 827, L31 